\title{
3D Gait Recognition based on Functional PCA on Kendall's Shape Space
}

\author{
Nadia Hosni ${ }^{\ddagger}$, Hassen Drira ${ }^{\ddagger}$, Faten Chaieb $^{\S}$ and Boulbaba Ben Amor ${ }^{\ddagger}$ \\ ¥IMT Lille Douai/CRIStAL (UMR CNRS 9189), University of Lille, France. \\ $\S$ Ecole Nationale des Sciences Informatique, CRISTAL/GRIFT, Manouba University, Tunisia.
}

\begin{abstract}
In this paper we propose a novel gait recognition approach from animated 3D skeletal data. Our approach is based on two disparate ideas from Shape Analysis and Functional Data Analysis (FDA) for a joint geometric-functional analysis. That is, skeletal sequences are viewed as time-parametrized trajectories on the Kendall's shape space when scaling, translation and rotation variations are filtered out from fixed-time 3D skeletons. A Riemannian Functional Principal Component Analysis (RFPCA) is carried out on our manifold-valued trajectories in order to build a new basis of principal functions, termed EigenTrajectories. Thus, each trajectory, could be projected into the eigenbasis which give rise to a compact signature, or EigenScores. The latter is fed to pre-trained 'One-vs-All' SVM classifiers for identity recognition and authentication. Based on the geometry of the underlying shape space, tools for re-sampling and synchronizing trajectories are naturally derived to apply the proposed variant of FPCA. We have conducted experiments on a subset of the CMU dataset. Our approach shows promising results compared to the state-of-the-art when a compact and robust signature is considered.
\end{abstract}

Keywords 3D gait recognition, Behavioral biometrics, Functional PCA, Kendall's trajectory, Riemannian geometry.

\section{INTRODUCTION}

Gait recognition is a relatively old research field in behavioral biometrics. Suitable properties as user-friendly, costeffective and non-invasive made it attractive in applications like crime investigation and physical access control. Over the past few years, several 2D methods have been proposed making use of the body either by establishing a prior body model [1] or by using the overall shape of silhouette attributes [2], [3]. However, the use of video data make the problems of self-occlusion, pose variations, illumination and appearance changes difficult to handle in addition to the important task of background subtraction [4]. In order to overcome these limitations, a new modality is emerging 3D skeletal data. Several approaches have been proposed recently by Ahmed et al. [5], Andersson et al. [6], Balazia and Sojka [7], Ball et al. [8] and Kwolek et al. [9] that exploit 3D skeletal data and have been tested on the output of MoCap (Motion Capture) sensors. This trend is increasingly confirmed with the development of affordable and reliable MoCap sensors (i.e. Kinect-like) and the availability of public development datasets [7]. Skeletal data are independent of illumination, robust to self-occlusion and pose variations. They are sources of anthropometric (body's height, length of arms, etc.) and kinematic features (evolution of angles, Center-ofMass trajectory, stride length, gait pattern, regularity, body's velocity, etc.). These features have been used separately or jointly in a set of recent approaches. For instance, Ahmed et al. [5] have computed horizontal (HDF) and vertical distances (VDF) between couples of joints. In [6], a gait descriptor was constructed by the mean and standard deviation of signals defined by the lower limbs angles (hips, knees and ankles). Kwolek et al. [9] proposed to compute bone rotations, interjoint distances, and the person's height whereas Preis et al. [10] extracted thirteen biometric features. Among them, eleven are static features of the human body (height, length of legs, length of both upper arms, etc.) and two are dynamic features that are step length and the body's speed. Dikovski et al. [11] used statistical tools such as the mean and the standard deviation on seven feature sets constructed from static body parameters during one gait cycle, joint angles and inter-joint distances. As for Sinha et al. [12], they proposed to combine many gait features like areas of upper and lower body, inter-joint distances along with the features proposed in [8] and [10]. In [7], authors focused on machine learning techniques in order to improve gait features classification results since they were largely used in the literature of 2D gait recognition. Thus, they have proposed to learn gait features that maximize the separability between the different classes of persons by a modification of Fisher's Linear Discriminant Analysis with Maximum Margin Criterion (MMC).

All the aforementioned methods deal with 3D joints of skeletons (or derived kinematics) in gait cycles. However, they ignore the functional structure of gait cycles since they are formed by skeletons differently shaped over the time and holding variations that can be discriminant to identify one walker by his walking way. Compared to these approaches, the present work considers these sequences as trajectories living in a certain space and parametrized by the time. Then, Functional Principal Component Analysis (FPCA) is used to create uncorrelated variables which could make identity classification from gait trajectories faster. To this end, two key preprocessing steps should be developed (1) normalize the temporal length of skeletal sequences, and (2) temporally align them to make inference interpretable. In summary, our approach maps skeletal sequences as time-parametrized trajectories on the Kendall's shape space. Grounding on the geometry of the space, tools for re-sampling and synchronizing trajectories are derived. Due to the orbit-manifold structure of the underlying space, an adapted variant of FPCA is proposed and applied to gait trajectories for identity recognition. Accordingly, the 
main contributions of this work are:

- Inspired from [13], a space-time representation of 3D gait-related skeletal sequences as trajectories on the Kendall's shape space where shape-preserving variations (scaling, translation and rotation) are filtered out.

- A variant of Functional PCA adapted to the manifoldvalued trajectories lying to the Kendall's shape space is proposed. It accounts for the non-linear structure and nontrivial geometry of the underlying space, as investigated very recently in [14] for more general Riemannian manifolds.

- A novel 3D gait recognition approach based on our variant of FPCA on the shape space. The proposed approach outperforms existing methods thanks to a joint geometric-functional analysis.

The rest of the paper is organized as follow. Section II presents how skeletal sequences are modeled on the Kendall's shape space as parametrized trajectories. It will remind briefly essential (Riemannian geometry) materials of the underlying space. In section III, we introduce our variant of FPCA on Kendall's shape space. Section IV describes our experimental setup and provides an evaluation of the proposed approach with respect to previous studies. Some conclusions and future perspectives are drawn in section $\mathrm{V}$

\section{Trajectories on Kendall's Shape Space}

Following the approach proposed in [13], 3D skeletal sequences are mapped to the Kendall shape space and thus viewed as time-parametrized trajectories. Accordingly, shapepreserving transformations such as translation, scaling and rotation are filtered out from the static representation [15]. Before coming to the main contribution of this paper, we first remind essential mathematical background on the geometry of this particular space.

\section{A. Riemannian Geometry of Kendall's shape space}

David G. Kendall defines the shape as all geometrical information that remains once transformations such us scaling, translation and rotation are filtered out [16]. In this work, we adopt the same theory of [15] to filter these shape-preserving transformations. Given a matrix representation $\tilde{X} \in \mathbf{R}^{n \times 3}$ of a 3D skeleton where $n$ is the number of 3D joints, we set $\sum_{i=1}^{n} \tilde{X}_{i, j}=0$, for $j=1,2,3$, then $\|\tilde{X}\|_{F}=1$, as follows. The product $X=H \tilde{X}$, where $H$ is Helmet sub-matrix sized $(n-1) \times n$, represents the centered coordinates of $\tilde{X}$ (as explained in [13]). With the condition $\|X\|_{F}=1$, the preshape space $\mathcal{C}=\left\{X \mid\|X\|_{F}=1\right\}$ is built. It is the unit sphere of $\mathbf{R}^{3(n-1)}$. To reach the shape space, rotations (elements of the special orthogonal group $S O(3)$, the $3 \mathrm{D}$ rotation group.) need to be filtered out by the definition of equivalent classes (or orbits), $[X]=\{X O \mid O \in S O(3)\}$, defined as all the rotated versions of $X$. So, the set of all $[X], X \in \mathcal{C}$ form the shape space $\mathcal{S}$ of $3 \mathrm{D}$ skeletons of $n$ joints. The tangent space attached to the preshape space at $X \in \mathcal{C}$ is given by
$T_{X}(\mathcal{C})=\left\{V \in \mathcal{C} \mid \operatorname{trace}\left(V^{T} X\right)=0\right\}$. Hence, the tangent plane to $\mathcal{S}$ at $[X]$ can be defined as,

$$
T_{[X]}(\mathcal{S})=\left\{V \in \mathcal{C} \mid \operatorname{trace}\left(V^{T} X\right)=0, \operatorname{trace}\left(V^{T} X A\right)=0\right\},
$$

where $A$ is any skew-symmetric matrix of size $3 \times 3$. The first condition makes $V$ tangential to the preshape space while the second condition imposes its orthogonality to the rotation orbit. Together, they guarantee $V$ to be tangent to $\mathcal{S}$. For convenience, the tangent space $T_{[X]}(\mathcal{S})$ is identified with $\mathbf{R}^{3 n-7}$. Considering the spherical structure of $\mathcal{C}$, it is possible to construct a geodesic (i.e. shortest) path along the space that connects a source shape $[X] \in \mathcal{S}$ to a target shape $[Y] \in \mathcal{S}$. It is given by the arc $[\alpha(t)]$ of the great circle going through $[X]$ and $[Y]$ as follows

$$
\alpha(t)=\frac{1}{\sin (\theta)}\left(\sin ((1-t) \theta) X+\sin (t \theta) Y O^{*}\right),
$$

where $\theta=\cos ^{-1}\left(\left\langle X, Y O^{*}\right\rangle\right)$ is length of the path $\alpha(t)$, called also geodesic distance and $O^{*}$ is the optimal rotation that optimally align $Y$ to $X$. Furthermore, the Exponential map and its inverse (Logarithm) map are defined by Eq.3 and Eq.4, respectively. Together, they allow to map data from the manifold to an arbitrary tangent space and inversely.

- Exponential map - for $V \in T_{[X]}(\mathcal{S})$, the Exponential function $\exp _{[X]}():. T_{[X]}(\mathcal{S}) \rightarrow \mathcal{S}$ is defined as

$$
\exp _{[X]}(V)=\left[\cos (\theta) X+\frac{\sin (\theta)}{\theta} V\right],
$$

where $\theta=\sqrt{\langle V, V\rangle}=\sqrt{\operatorname{trace}\left(V V^{T}\right)}$.

- Logarithm map - the inverse of the Exponential map $\exp _{[X]}^{-1}():. \mathcal{S} \rightarrow T_{[X]}(\mathcal{S})$, is given by $V$

$$
V=\exp _{[X]}^{-1}([Y])=\frac{\theta}{\sin (\theta)}\left(Y O^{*}-\cos (\theta) X\right)
$$

Now that we have set all materials for static skeletal shape analysis, we consider an arbitrary skeletal sequence as a timeparametrized trajectory on the shape space $\mathcal{S}$. Our aim is to adapt the well-known Functional PCA to trajectories on $\mathcal{S}$ and thus create new uncorrelated variables to represent them. In addition to the difficulty related to the structure complexity (non-linearity) of $\mathcal{S}$, trajectories should be aligned. In other words, the effect of different time-rate and the divergence in trajectory's starting shape should be filtered out. This problem is well-known in functional data analysis by registration problem. Finally, trajectories should have the same temporal length. We present solutions to the last two problems in the next section. Our variant of FPCA applied to our orbit-manifoldvalued trajectories is presented in Section III.

\section{B. Trajectories on the Kendall's Space}

Actually, the segmentation of gait sequences results to cycles, termed strides. They have different temporal length i.e. different number of skeleton frames per sequence. In order to apply FPCA on shape trajectories as it will be 
explained in Sec.III, two conditions are required - (1) lengths of trajectories must be equal; and (2) they must be temporally aligned. To this end, a re-sampling step of the sequences on $\mathcal{S}$ is applied based on the geodesic path given by Eq. 2 as a natural interpolation tool in the shape space $\mathcal{S}$. In addition a temporal alignment step of shape trajectories is performed using a proper elastic metric defined in [13] on Kendall's shape space. These points are deeply described in the following items.

Re-sampling trajectories on $\mathcal{S}$ : here, we explain the resampling of a discrete trajectory $\alpha(t)_{t=t_{1}, . ., t_{n}}, \alpha\left(t_{i}\right) \in \mathcal{S}$ of length $n$ in order to get a new version $\alpha(s)_{s=s_{1}, \ldots, s_{m}}$ of length $m$ (either $m<n$ or $m>n$ ). This step allows obtain fixedlength shape trajectories. if $m>n$, we have increased the temporal resolution and this action is known as up-sampling. Otherwise, it is a down-sampling. The resulting trajectory $\alpha(s)$ is given by $\alpha\left(s_{i}\right)=\frac{1}{\sin (\theta)}\left(\sin \left(w_{2} \theta\right) x+\sin \left(w_{1} \theta\right) y\right)$ where $w_{1}=\frac{s_{i}-t_{i 1}}{t_{i 2}-t_{i 1}}, w_{2}=\frac{t_{i 2}-s_{i}}{t_{i 2}-t_{i 1}}$ and $s_{i}$ verifies $t_{i_{1}} \leq s_{i} \leq t_{i_{2}}$. Clearly, we used here the interpolation tool defined in Eq.2 which allows to connect successive shapes in the trajectory based on the geodesic (shortest) path between them.

Temporal alignment of trajectories on $\mathcal{S}$ : given the shape trajectories as functional data, they might not be comparable since each of them has its pace and gait velocity. In other words, there is no correspondence between functions to be compared. This problem is known in Functional Data Analysis as registration problem. This is why a synchronization is a must so that FPCA is well interpretable. As a solution to this issue, DTW (Dynamic Time Warping) algorithm is quite used. The goal here is to find the optimal registration $\gamma^{*}$ that minimizes the distance $d\left(\alpha_{1}, \alpha_{2}\right)=\operatorname{argmin}_{\gamma} \int_{0}^{T} d_{s}\left(\alpha_{1}(t), \alpha_{2}(\gamma(t))\right) d t$ between two trajectories $\alpha_{1}$ et $\alpha_{2}$. However, this cost function is not a proper metric, since the alignment of $\alpha_{1}$ to $\alpha_{2}$ is not the same as the alignment of $\alpha_{2}$ to $\alpha_{1}$ (i.e. not symmetric). In [13], Ben Amor et al. solved this problem by defining Transported-SRVF, which is a representation of a trajectory on the tangent space to $\mathcal{S}$, attached to a reference point $[R]$ of the shape space $\mathcal{S}$, as well as an elastic metric that is interesting mathematically and computationally. In other words, they represent trajectories on Riemannian manifolds such that the distance between two trajectories is invariant to identical time warping. We have adopted the latter approach (i.e. T-SRVF)for temporal registration of gait trajectories.

\section{FPCA ON KENDALL TRAJECTORIES}

Previously, we have introduced mathematical materials and preprocessing tools, essential to apply FPCA on gait trajectories. Here, we introduce the main contribution of the paper, i.e. our Functional PCA variant on Kendall's shape space. We first review briefly Functional Principal Component Analysis (FPCA) in Euclidean spaces, then, we present our variant adapted to the Kendall's shape space.

\section{A. Functional PCA in Euclidean Spaces}

Functional Principal Components Analysis (FPCA) is a powerful statistical tool used to capture variations of random functions in terms of principal modes of variations, while reducing the dimensionality (i.e create new uncorrelated variables with maximum variance) [17]. That is, FPCA represents any random function in a new function basis of the covariance operator of a set functions. More formally, given a stochastic square integrable process $X(t)$, where $t \in \mathcal{T}$, the mean is first defined by $\mu(t)=\mathrm{E}(X(t))$ and the covariance operator is given by $G(s, t)=\operatorname{Cov}(X(s), X(t))=\sum_{k=1}^{\infty} \lambda_{k} \varphi_{k}(s) \varphi_{k}(t)$, $t, s \in \mathcal{T}$ where $\lambda_{1} \geq \lambda_{2} \geq \cdots \geq 0$ are the eigenvalues and $\varphi_{k}$ are the orthogonal principal functions of the linear HilbertSchmidt operator. Grounding on the Karhunen-Loeve theorem, it is possible to express the centered process $X(t)-\mu(t)$ using the principal functions basis as $X(t)-\mu(t)=\sum_{k=1}^{\infty} \xi_{k} \varphi_{k}(t)$, where $\xi_{k}=\left\langle X(t)-\mu(t), \varphi_{k}(t)\right\rangle=\int_{\mathcal{T}}(X(t)-\mu(t)) \varphi_{k}(t) d t$ is the eigenvalue corresponding to the $k^{\text {th }}$ eigenfunction, $\varphi_{k}$ (here, $\langle.$,$\rangle is the inner product of the ambient L^{2}$ Hilbert space of valued square integrable functions). The centered process is then equivalent to the coefficients $\xi_{1}, \xi_{2}, \cdots$. A $K$-truncated version is given by a linear combination of the $K$ first principal functions once the mean value is subtracted as,

$$
X(t) \approx \tilde{X}_{K}(t)=\mu(t)+\sum_{k=1}^{K} \xi_{k} \varphi_{k}(t) .
$$

Coming back to our trajectories living on Kendall's shape space, the set of orbits of the unit sphere of $\mathbf{R}^{3(n-1)}$ under the group action of the special orthogonal group $S O(3)$. Several challenges encounter the adaptation of the conventional FPCA to our trajectories. One needs first to define the 'mean' trajectory $\mu(t)$ on the shape space $\mathcal{S}$. Once done, the linear combination $\sum_{k=1}^{K} \xi_{k} \varphi_{k}(t)$ in Eq.5 is no more valid, as the approximation $\tilde{X}(t)$ do not lie necessarily to $\mathcal{S}$. In the following items, we propose an intrinsic method to compute a sample average trajectory of a set of gait trajectories, then, we present our variant of FPCA adapted to the underlying space.

\section{B. Computing an Average Sample Trajectory on $\mathcal{S}$}

Computing statistical summaries on shape trajectories is not trivial as several requirements should be fulfilled. First, trajectories should have the same temporal length. Second, gait trajectories exhibits temporal variability due to the difference in the execution rate and eventually different starting shape. To solve these problems, we use the pre-processing solutions presented in Section II-B. As a result, we build a set of $N$ synchronized gait trajectories $\left\{\alpha_{j}(t)_{t=1, . ., n}\right\}_{1 \leq j \leq N}$ of length $n$. We then compute an average trajectory by computing cross-sectional Karcher mean [15] defined by, $\hat{\mu}(t)=$ $\operatorname{argmin}_{[X] \in \mathcal{S}} \sum_{i=1}^{N} d_{\mathcal{S}}\left([X],\left[X_{i}\right]\right)^{2}$, at each time $t$. That is, the problem of computing an average trajectory turns to estimate a Karcher sample mean at each time $t$, on synchronized and re-sampled trajectories. Algorithm.1 summarizes the iterative procedure to compute cross-sectional Karcher means and its extension to trajectories. Figure.1 illustrates a sample average 
trajectory of a collection of several hundred of gait cycles related to 54 different subjects. Despite huge number of samples, their average trajectory exhibits natural deformations of a gait cycle. This is due to both the preprocessing steps (i.e. re-sampling and temporal alignment) and the cross-sectional Karcher mean estimation procedure in the shape space.

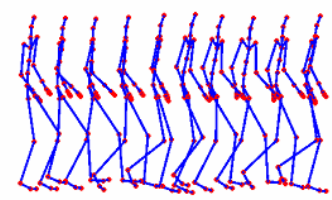

Fig. 1. Equally-spaced shapes of the sample average trajectory computed over a collection of several hundred of gait cycles of 54 different subjects.

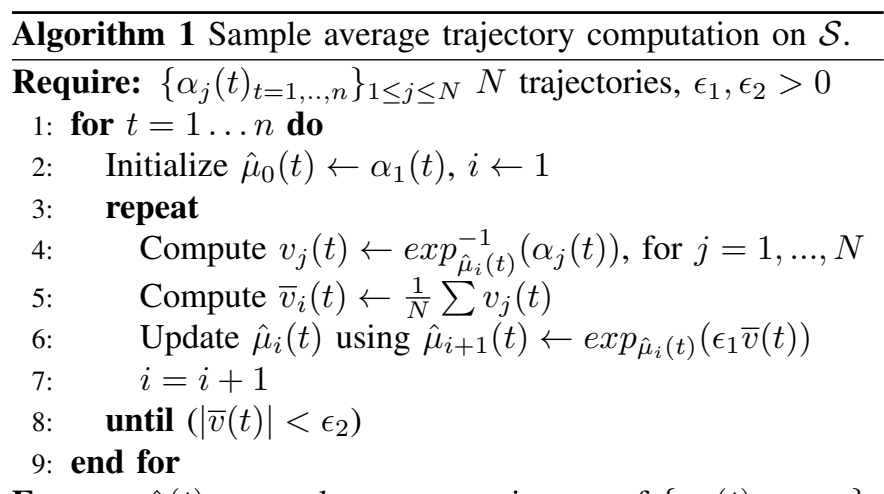

Now, one can move to the core of the approach which consists in performing Functional PCA on gait shape trajectories.

\section{Functional PCA over trajectories on $\mathcal{S}$}

As has been explained previously, the application of FPCA to gait shape trajectories isn't straightforward. This is mainly due to the non-linear (spherical) geometry of the pre-shape space $\mathcal{C}$ and the orbit-manifold structure of the shape space. Based on the very recent work [14], we propose here an intrinsic approach to perform FPCA on the set of tangent spaces attached to $\mathcal{S}$ at $\hat{\mu}(t)$, the average trajectory. In fact, given $N$ trajectories $\left\{\alpha_{j}(t)_{t=1, . ., n}\right\}_{1 \leq j \leq N}$ and their average trajectory $\hat{\mu}(t)_{t=1, \ldots, n}$, they are log-mapped to the tangent spaces $T_{\hat{\mu}(t)}(\mathcal{S})$ using Eq.4 which gives $V(t)$. Since tangent spaces $T_{\hat{\mu}(t)}(\mathcal{S})$ are identified with $\mathbb{R}^{3 n-7}, V(t)$ could be viewed as discrete versions of random functions of $L^{2}\left(\mathbb{R}^{3 n-7}\right)$, the Hilbert space of valued square integrable functions of $\mathbb{R}^{3 n-7}$. As a result, Functional PCA on $\mathcal{S}$ corresponds to a multivariate FPCA in $\mathbb{R}^{3 n-7}$. Thus, $\mathrm{V}(\mathrm{t})$ and $\mathrm{X}(\mathrm{t})$ are expressed as follows:

$$
\left\{\begin{array}{l}
V(t) \approx \tilde{V}_{K}(t)=\hat{\mu}(t)+\sum_{k=1}^{K} \xi_{k} \varphi_{k}(t) \\
X(t) \approx \tilde{X}_{K}(t)=\exp _{\hat{\mu}(t)}(V(t)) .
\end{array}\right.
$$

Recently, in [14], a more general Riemannian FPCA have been proposed. However, to our knowledge, this is the first formulation of FPCA on Kendall's shape space. Algorithm.2 summarizes the steps of our intrinsic adaptation of PCA to Kendall's shape trajectories.

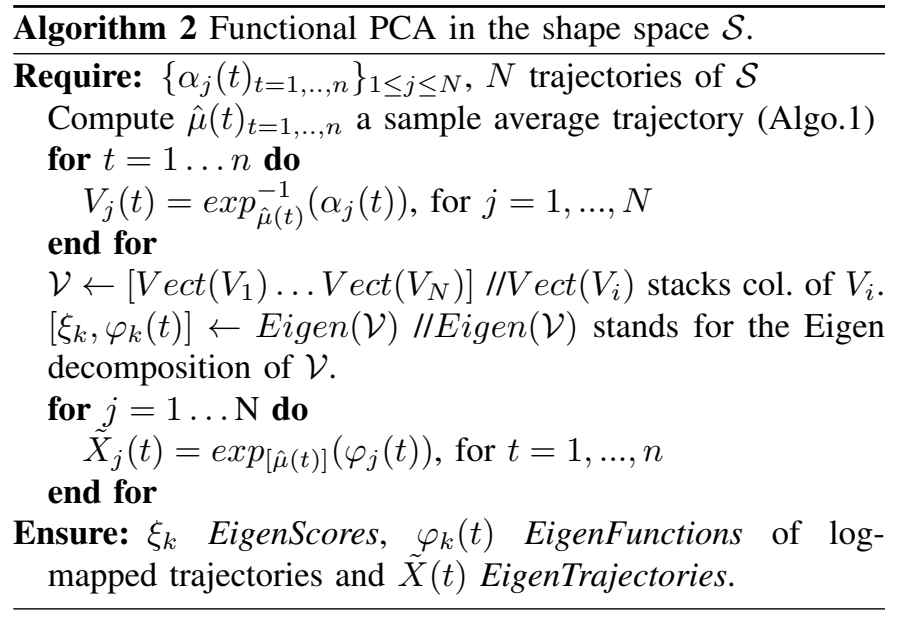

To illustrate this idea, we provide in Figure. 2 the first four modes of variation (i.e. EigenTrajectories) computed on a set of gait trajectories. The first principal component is in the direction of the largest spread (or variance) and so on. These EigenTrajectories represent gait sequences once associated EigenFunctions are projected back to the shape space.
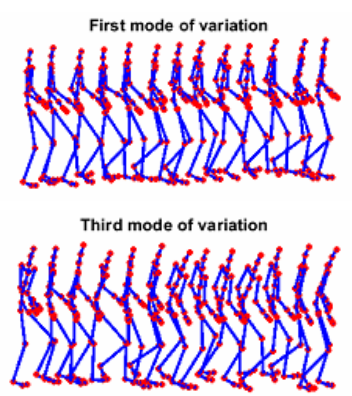

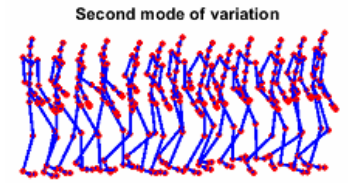

Fourth mode of variation

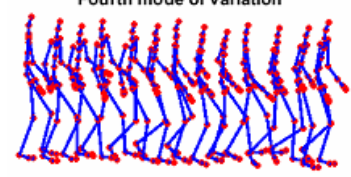

Fig. 2. Four first EigenTrajectories outputs of Algo.2 on a set of several hundred trajectories of 54 subjects.

Now, given an arbitrary gait trajectory (even never seen by FPCA), it is easy to compute its new coordinates in the basis of EigenTrajectories and reconstruct it back in the Kendall shape space for the purpose of comparison. Different steps of the projection and reconstruction are enumerated in Algo.3. An example of reconstruction is illustrated in Figure.3. The figure shows the good reconstruction achieved. The original gait trajectory and its reconstructed version are perfectly synchronized.

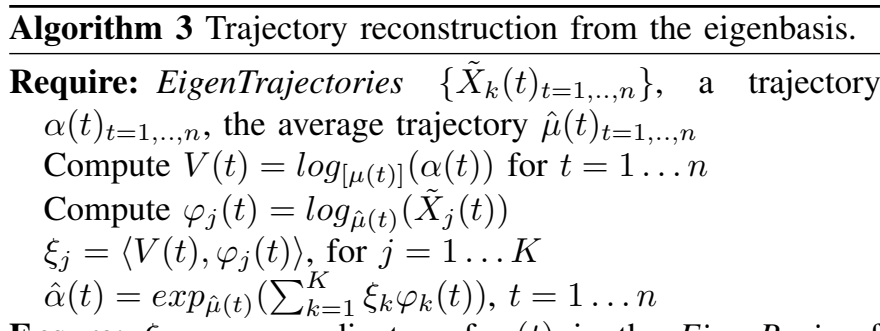

Ensure: $\xi_{k}$ new coordinates of $\alpha(t)$ in the EigenBasis of FPCA and $\hat{\alpha}$ the reconstruction of $\alpha$. 


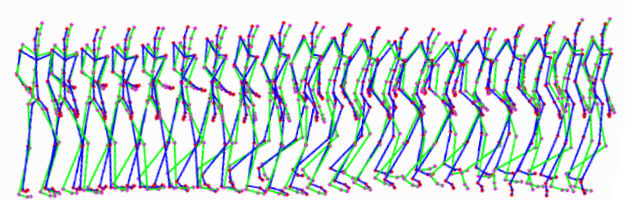

Fig. 3. One gait trajectory (blue) and its reconstruction (green) using Algo.3.

\section{EXPERIMENTAL Results}

To demonstrate the effectiveness of the proposed approach, we perform gait recognition on MoCap skeletons sequences following the experimental setting described in [7]. We report results for the recognition and authentication scenarios. We carried experiments on a subset of the well-known Mocap CMU dataset ${ }^{1}$. This dataset contains different human actions and daily activities such as walking, running, playing tennis, etc. It includes several gait-related sequences as well of 54 subjects. The authors of [7] have extracted gait cycles and have released them ${ }^{2}$. In total, they extracted more than 3,800 sequences of the 54 subjects. Based on their experimental setup, a 3-fold cross validation is performed that consists to split extracted gait cycles to three folds: one training set and two evaluation sets in a manner that each set contains disjoint instances from all the classes of subjects. In our experiments, the FPCA basis is formed using data of training set (Algo.2), and the evaluation data are projected into the EigenBasis (Algo.3). The classification is performed using 'One-vs-All' classifiers using a nested 10 fold cross validation by considering one fold as a test set and the nine others as gallery set.

The evaluation metrics such as CRR, AUC and MAP are used to illustrate the performance of the proposed approach.

In the following, we briefly remind these metrics,

- The CRR i.e. the average Correct Classification Rate is the ratio of correctly predicted observations to the total observations.

- ROC curve is the Receiver Operating Characteristic curve. It shows the variation of the True Positive Rate (TPR) in function of the False Positive Rate (FPR).

- The AUC score (Area Under Curve) measures the area under the ROC curve.

- The Recall is the ratio of correctly predicted positive observations to the all observations whereas the Precision is the ratio between correctly predicted positive observations and all the positive observations predicted.

- the MAP score (Mean Average Precision) is the area under the Recall/Precision graph that makes a classifier good if its Precision will stay high as Recall increases.

The values of the evaluation metrics are reported in Table I together with the dimension of the features. Comparative results of state-of-the-art methods are also reported. The

\footnotetext{
${ }^{1}$ http://mocap.cs.cmu.edu

${ }^{2}$ https://gait.fi.muni.cz/ $\$ framework
}

TABLE I

COMPARISON WITH THE STATE-OF-ART BASED ON THE RESULTS REPORTED IN [7].

\begin{tabular}{c|c|c|c|c} 
Method & Dimension & CRR & AUC & MAP \\
\hline Ahmed et al. [5] & 24 & 0.7134 & 0.6387 & 0.1617 \\
Andersson et al. [6] & 80 & 0.7787 & 0.6545 & 0.1926 \\
Ball et al. [8] & 18 & 0.6963 & 0.6612 & 0.1454 \\
Dikovski et al. [11] & 71 & 0.8926 & 0.6964 & 0.2582 \\
Kwolek et al. [9] & 660 & 0.9099 & 0.6477 & 0.2121 \\
Preis et al. [10] & 13 & 0.13 & 0.6236 & 0.0579 \\
Sinha et al. [12] & 45 & 0.7666 & 0.6809 & 0.1858 \\
Balazia et al. [7] & & 0.8314 & 0.6216 & 0.1643 \\
(PCA+LDA) $^{\text {between 54 }}$ & & & \\
Balazia et al. [7] & and $N_{L}^{2}-54$ & & & \\
(MMC) $^{\text {Ours (80\%) }}{ }^{3}$ & 53 & 0.9102 & 0.7551 & 0.2996 \\
Ours (98\%) & & & & \\
\hline
\end{tabular}

${ }^{1}$ Number of subjects in the extracted Database.

2 Number of labeled templates for learning the features.

${ }^{3}$ Percentage of variance explained by the $r$-first EigenTrajectories.

identification metric evaluation's results indicates that our approach outperforms existing methods in terms of CRR. Actually, when using $98 \%$ of explained variance in FPCA, we report $92.23 \%$ correct classification and this represents the best CRR compared to the previous methods. The feature vector dimension is comparable to the size of feature vectors of previous approaches. When only $80 \%$ of the energy is used, the feature vector is very small (of size in average 14) and the CRR is $74.69 \%$. For deeper understanding of the impact of the low-dimensionality in the application of FPCA, we report in Table II the evolution of CRR in terms of the amount of energy used while performing FPCA. We notice that $90 \%$ of the energy at least is needed to perform competitive results with state-of-the-art. In the third column of Table I, we report the MAP that represents the area under the Rappel-Precision curve. The results reported by the proposed approach is higher compared to the state-of-the-art. Even with $80 \%$ of the energy, the reported MAP $(47.09 \%)$ remains considerably better than the best result in state-ofthe-art $29.96 \%$. The corresponding Recall-Precision curve is reported in Figure. 4 at the right and is above the state-ofthe-art ones. The difference in performance is considerable implying that the combination between the One-vs-All SVMs and FPCA results in an efficient gait recognition system.

Again, this difference is actually confirmed by the ROC curves reported in Figure. 4 (in the left panel), together with AUC value in Table I. In practice, the ROC curves and the recall-Precision curves relative to the proposed approach were computed by averaging the 54 curves resulting for each test set when performing the 10 -fold cross-validation. These computations are based on the predicted probabilities returned by each learned SVM Model (for each class) when using the One-vs-All SVM classification schema with 54 classes. We note that where the majority of existing works use distancebased approaches, in our approach subject-specific classifiers are trained using SVM models. 

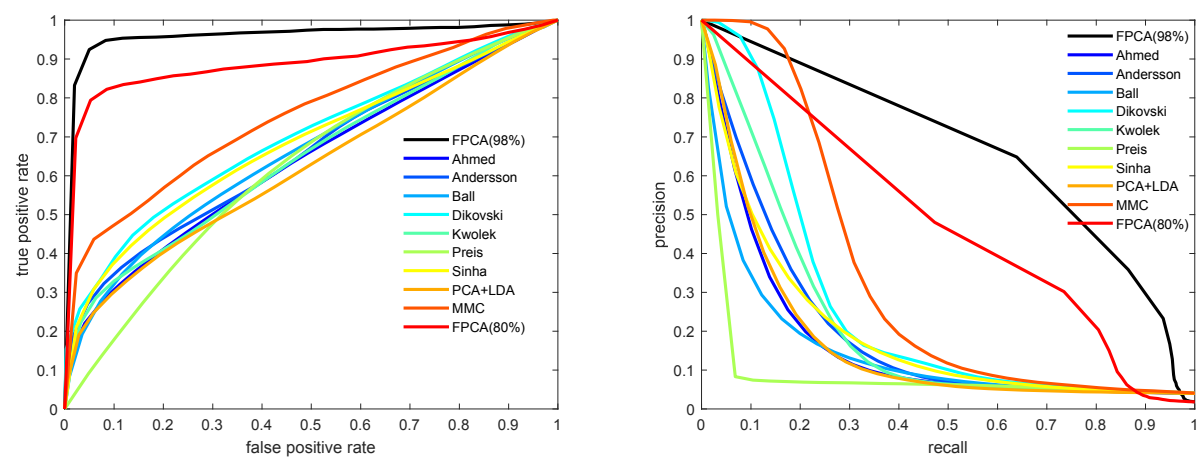

Fig. 4. Reported ROC (left) and Recall-Precision (right) curves of the proposed approach at $80 \%$ and $98 \%$ of explained variance.

These results demonstrate that our variant of Functional PCA adapted to the Kendall's shape space is able to capture a better geometric-functional signature of the human body. The signature reflects higher discriminative power with respect to previous methods. All that being said, we can not deny that there are many mis-classifications that occur using our method that is maybe due to the Reconstruction errors as the projection on the eigen-basis results sometimes is non-accurate coefficients.

TABLE II

EVOLUTION OF THE CRR WHEN VARYING THE PERCENTAGE OF VARIANCE EXPLAINED BY THE $r$-FIRST EIGENTRAJECTORIES.

\begin{tabular}{c|c|c|c|c|c|c} 
\% of variance & $70 \%$ & $75 \%$ & $80 \%$ & $85 \%$ & $90 \%$ & $\mathbf{9 8 \%}$ \\
\hline CRR & 0.677 & 0.715 & 0.746 & 0.790 & 0.831 & $\mathbf{0 . 9 2 2}$
\end{tabular}

\section{CONCLUSION AND FUTURE WORK}

In this paper, we have introduced a joint geometricfunctional analysis approach of 3D skeletal data for the purpose of gait recognition. We have proposed a variant of FPCA adapted to the Kendall's shape space able to create new uncorrelated variables for gait trajectory representation. Through the experiments conducted on a subset (limited to gait) of the CMU dataset and following the experimental settings of the state-of-the-art, we have demonstrated the effectiveness of the proposed approach. The proposed framework is generic enough to be extended to other future directions including (1) gait classification for preventing fall of elderly; (2) performing statistical $t$-tests on obtained eigenScores to study the effects of diseases as autism, perkinson, or simply the age or the athletic history on our daily activities as (gait, sit-to-stand, etc.).

\section{ACKNOWLEDGMENT}

This work received the financial support of the PHC Utique program for the DEFI project \#16G1403.

\section{REFERENCES}

[1] J. Man and B. Bhanu, "Individual recognition using gait energy image," IEEE transactions on pattern analysis and machine intelligence, vol. 28, no. 2, pp. 316-322, 2006.

[2] L. Wang, T. Tan, H. Ning, and W. Hu, "Silhouette analysis-based gait recognition for human identification," IEEE transactions on pattern analysis and machine intelligence, vol. 25, no. 12, pp. 1505-1518, 2003.
[3] D. Tao, X. Li, X. Wu, and S. J. Maybank, "General tensor discriminant analysis and gabor features for gait recognition," IEEE Transactions on Pattern Analysis and Machine Intelligence, vol. 29, no. 10, 2007.

[4] J. Sun, Y. Wang, and J. Li, "Gait recognition," in Motion Tracking and Gesture Recognition, C. M. Travieso-Gonzalez, Ed. Rijeka: InTech, 2017, ch. 07. [Online]. Available: http://dx.doi.org/10.5772/68119

[5] M. Ahmed, N. Al-Jawad, and A. T. Sabir, "Gait recognition based on kinect sensor," in Real-Time Image and Video Processing 2014, vol. 9139. International Society for Optics and Photonics, 2014, p. 91390 B.

[6] V. O. Andersson and R. M. Araujo, "Person identification using anthropometric and gait data from kinect sensor," in Proceedings of the Twenty-Ninth AAAI Conference on Artificial Intelligence, ser. AAAI'15. AAAI Press, 2015, pp. 425-431. [Online]. Available: http://dl.acm.org/citation.cfm?id=2887007.2887067

[7] M. Balazia and P. Sojka, "Learning robust features for gait recognition by maximum margin criterion," in Pattern Recognition (ICPR), 2016 23rd International Conference on. IEEE, 2016, pp. 901-906.

[8] A. Ball, D. Rye, F. Ramos, and M. Velonaki, "Unsupervised clustering of people from 'skeleton' data," in Proceedings of the Seventh Annual ACM/IEEE International Conference on Human-Robot Interaction, ser. HRI '12. New York, NY, USA: ACM, 2012, pp. 225-226. [Online]. Available: http://doi.acm.org/10.1145/2157689.2157767

[9] B. Kwolek, T. Krzeszowski, A. Michalczuk, and H. Josinski, 3D Gait Recognition Using Spatio-Temporal Motion Descriptors”, bookTitle ="Intelligent Information and Database Systems: 6th Asian Conference, ACIIDS 2014, Bangkok, Thailand, April 7-9, 2014, Proceedings, Part II. Cham: Springer International Publishing, 2014, pp. 595-604.

[10] J. Preis, M. Kessel, M. Werner, and C. Linnhoff-Popien, "Gait recognition with kinect," 012012.

[11] B. Dikovski, G. Madjarov, and D. Gjorgjevikj, "Evaluation of different feature sets for gait recognition using skeletal data from kinect," Information and Communication Technology, Electronics and Microelectronics (MIPRO), 2014 37th Inter, May 2014.

[12] A. Sinha, K. Chakravarty, and B. Bhowmick, "Person identification using skeleton information from kinect," in In The Sixth International Conference on Advances in Computer-Human Interactions, pp. 101-108.

[13] B. Ben Amor, J. Su, and A. Srivastava, "Action recognition using rate-invariant analysis of skeletal shape trajectories," IEEE Trans. Pattern Anal. Mach. Intell., vol. 38, no. 1, pp. 1-13, 2016. [Online]. Available: https://doi.org/10.1109/TPAMI.2015.2439257

[14] X. Dai and H.-G. Müller, "Principal component analysis for functional data on riemannian manifolds and spheres," arXiv preprint arXiv:1705.06226, 2017.

[15] I. Dryden and K. Mardia, Statistical shape analysis. Wiley, 1998.

[16] D. G. Kendall, "Shape manifolds, Procrustean metrics, and complex projective spaces," Bulletin of the London Mathematical Society, vol. 16, no. 2, pp. 81-121, 1984. [Online]. Available: http: //blms.oxfordjournals.org/content/16/2/81.abstract

[17] M. Jones and J. A. Rice, "Displaying the important features of large collections of similar curves," The American Statistician, vol. 46, no. 2, pp. 140-145, 1992. 